\title{
Research Paper \\ The Relationship between Disability and Variables of Depression, Cognitive Status, and Morale among Older People
}

\author{
Mohammad Reza Shahbazi ${ }^{1},{ }^{*}$ Mahshid Foroughan ${ }^{2}$, Reza Salman Roghani ${ }^{3}$, Mehdi Rahgozar ${ }^{4}$
}

1. Department of Ageing, University of Social Welfare and Rehabilitation Sciences, Tehran, Iran

2. Iranian Research Center on Ageing, University of Social Welfare and Rehabilitation Sciences, Tehran, Iran.

3. Department of Clinical Sciences, University of Social Welfare and Rehabilitation Sciences, Tehran, Iran.

4. Department of Statistics, University of Social Welfare and Rehabilitation Sciences, Tehran, Iran.

Citation: Shahbazi MR, Foroughan M, Salman Roghani MR, Rahgozar M. [The relationship between disability and depression, cognitive status and morale among older persons (Persian)]. Iranian Journal of Ageing. 2016; 11(4):132-141. http://dx.doi.org/10.21859/sija-1101132

: http://dx.doi.org/10.21859/sija-1101132

Received: 08 Nov. 2015 Accepted: 04 Feb. 2016

Key words: Cognition, Depression, Disability, Morale, Older adults, World health organization

\begin{abstract}
Objectives This study aimed to assess disability and its relationship with depression, cognitive status, and morale in older people.

Methods \& Materials This descriptive-analytic research was conducted in a day-care rehabilitation center of Kahrizak charity foundation. A total of 90 older adults were selected by total enumeration sampling method. For the evaluation of study participants, we used The World Health Organization Disability Assessment 2.0 (WHODAS II) (36 items). This questionnaire examines the person's disability in 6 domains of cognition, mobility, self-care, getting along, life activities, and social participation. The depression status was determined by Geriatric Depression Scale (GDS-15), cognitive status by Abbreviated Mental test (AMT), and morale of older people by Lawton's Philadelphia Geriatric Center Morale Scale. The Chisquare test, Independent t-test, Mann-Whitney U-test, Kruskal-Wallis, and the Spearman correlation coefficient were carried out to analyze the data.

Results The mean (SD) score of older adults' disability was 20.61 (13.66) indicating a significant difference between men and women ( $P=0.001)$. The women's mean disability score was higher than that of men. With regard to 6 domains of disability, the highest disability was seen in domains of mobility, life activities, and participation. There were also significant differences between men and women with regard to the mean disability scores of mobility $(P=0.001)$, life activities $(P=0.001)$, and participation $(P=0.005)$, i.e., the mean disability scores of women were higher than those of men. However, there were no differences between men and women with regard to domains of getting along, cognition, and self-care. Furthermore, there were no significant differences between various educational groups with regard to mean scores of disabilities. There were significant associations between disability scores and depression $(P<0.001)$, cognitive status $(P<0.001)$, and morale of older people $(P<0.001)$. This association was direct in depression and reverse in cognitive status, and morale, i.e., with an increase in depression scores and a decrease in cognitive status and morale, the disability of older people increases. There were significant and reverse associations between disability and 3 subscales of spirit of agitation $(P<0.001)$, attitudes to aging $(P<0.001)$, and dissatisfaction with loneliness $(P<0.001)$.

Conclusion Disability in older people had a significant relationship with their depression, cognitive status, and morale. Thus, the degree of their disability can be lowered by prevention and early treatment of depression, promotion of memory, delaying cognitive disorders, as well as providing morale enhancement programs, creating a positive attitude toward old age, and increasing life satisfaction in older people.
\end{abstract}

* Corresponding Author:

Mahshid Foroughan, PhD

Address: Iranian Research Center on Ageing, University of Social Welfare and Rehabilitation Sciences, Kodakyar Ave., Daneshjo Blv., Evin, Tehran, Iran. Tel: +98 (21) 2280004

E-mail: m_foroughan@yahoo.com 


\title{
ارتباط ناتوانى با افسردَّى، وضعيت شناختى و روحيه در سالمندان
}

\author{
محمدرضا شهبازى'، "مهشيد فروغان ب، رضا سلمان روغنى"، مهدى رهكذر" \\ 1 - كروه سالمندي، دانشعاه علوم بهزيستى و توائبخشى، تهرانء ايران.

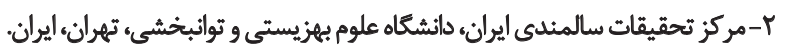

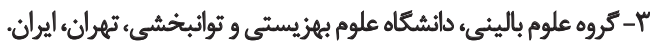

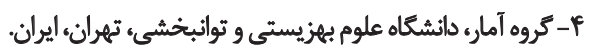

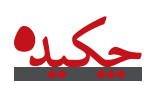

هدالق هدف اين مطالعه بررسى ميزان ناتوائى و ارتباط آن با افسردكى، اختلال شئاخت و روحيه در سالمندان بود.

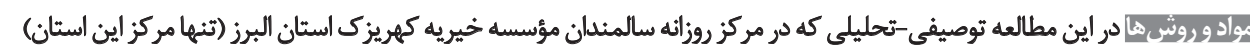

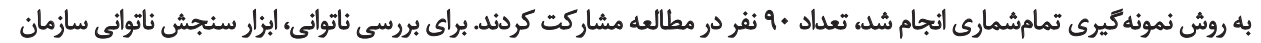

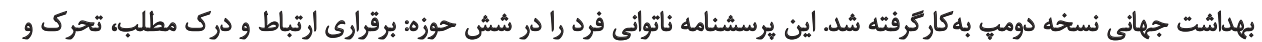

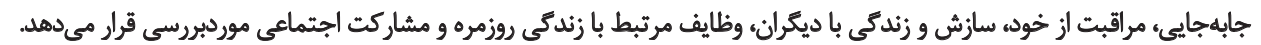

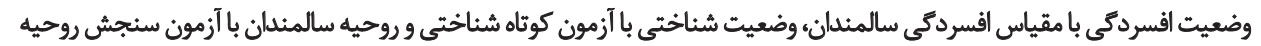

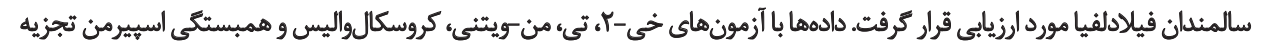
وتحليل شد. يافتله ها ميانكين نمره ناتوانى سالمندان

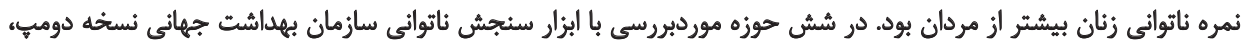

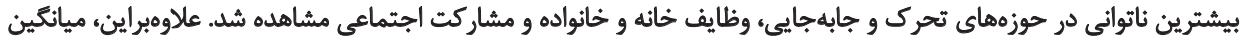

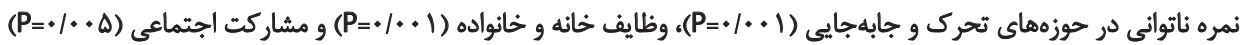

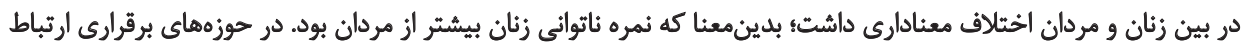

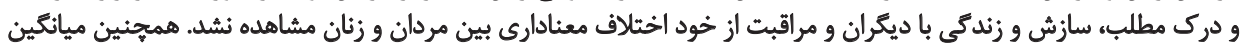

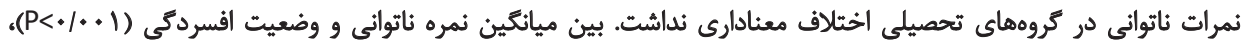

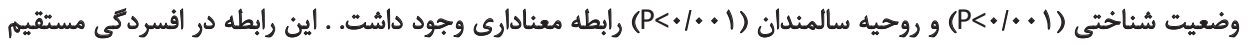

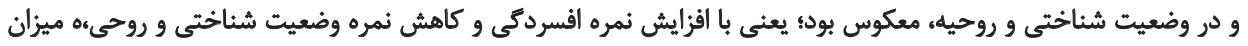

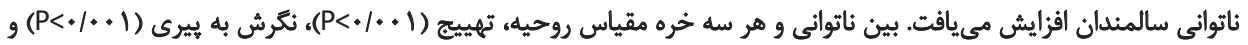

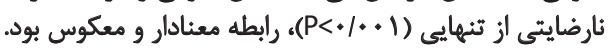

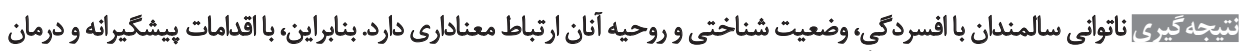

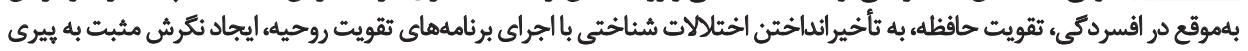

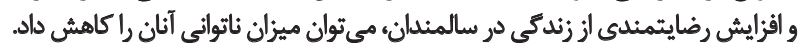

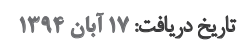
تاريخ بذيرش: 14 يهمن

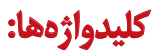

افسر كمىي، روحيه، سازمان

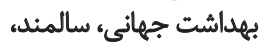
شئاخت، ناتواني

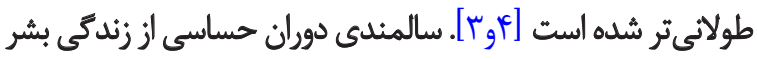

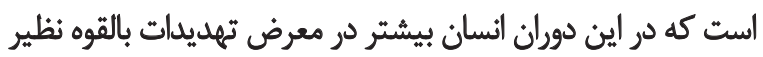

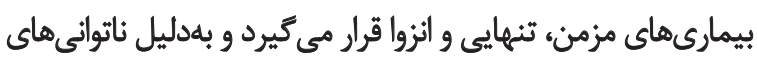

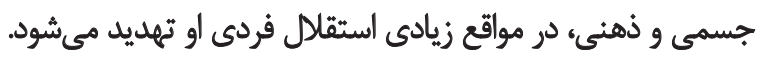
براساس تعريف سازمان جهانى بهداشت در دستهبندى ICF '، '، ناتوانى

1. International Classification of Functioning, Disability and Health سالمندى را مى توان دورهاى از زندگى ناميد كه تمامى موجودات

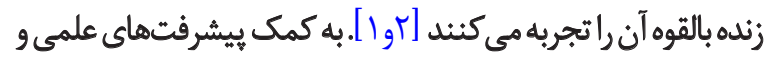

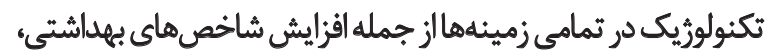

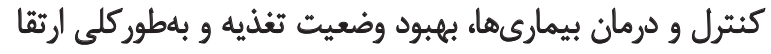

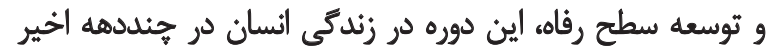

: نويسئده مسئول:

دكتر مهشيد فروغان نشانى: تهران، اوين، خيابان دانشجو، بن فيست كوديار، دانشكاه علوم بهزيستى و توانبخشى، مركز تحقيقات سالمثلى ايران.

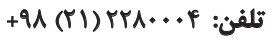
بـ_foroughan@yahoo.com: بلكت الكترونيكى 
كاهش رضايتمندى از زندكى و كاهش كيفيت زندكّى افراد را بهدئبال

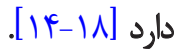

بعضى از مطالعات ارتباط معنادارى بين ناتوانى با وضعيت سلامت

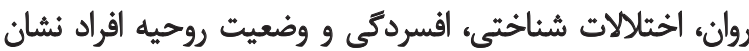

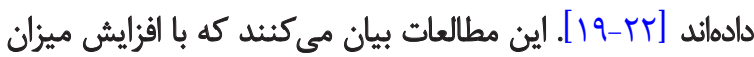

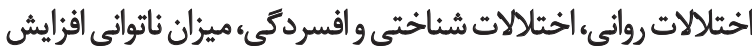

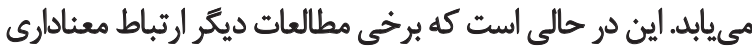

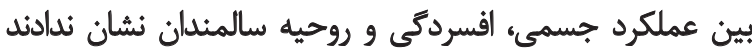

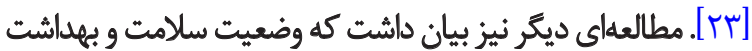

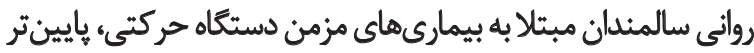
از وضعيت روانى سالمندانى است كه مبتلا به بيمارى هانى حركني دينى

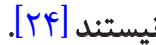

مطالعات مذكور نشان مي دهند كه هنوز دربارهارتباط متقابل ناتوانى ني

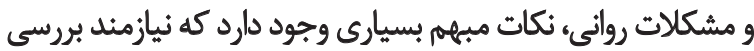

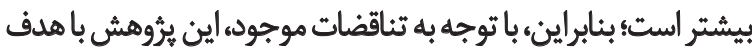

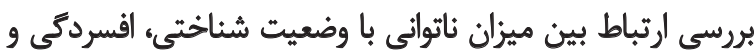

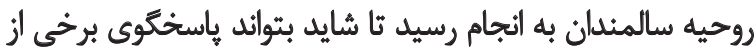

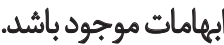

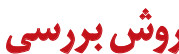

اين مطالعه كه در سال سوهו انجام شده يك يرؤهش مقطعى

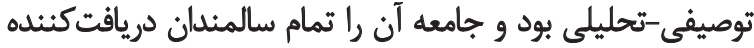

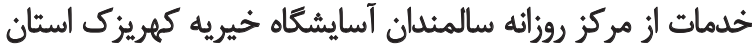

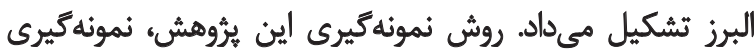

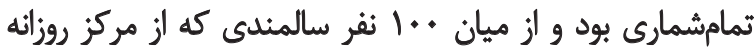

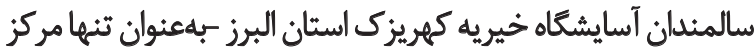

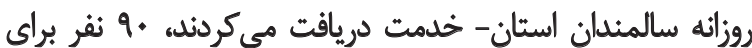

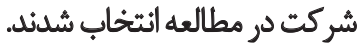

از جمله شرايط ورود به مطالعه، اعلام رضايت كتبى براي شركت

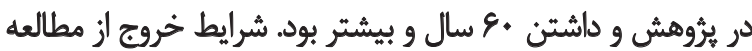

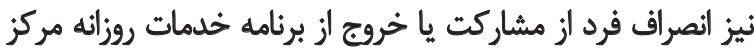

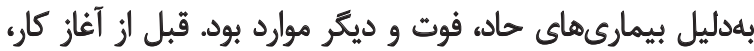

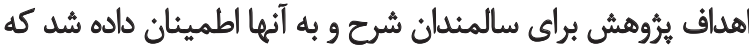

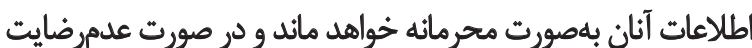

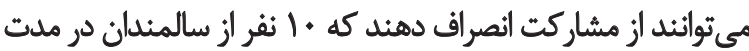
زمان يُروهش از شركت در مطالعه انصراف دادند.

ابزار مطالعه براى سنجش ناثوانى سالمندان، نسخه عب سؤالى

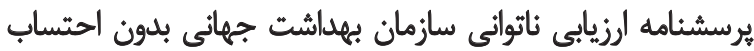

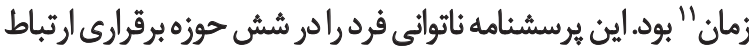

11. WHODAS II-36 items without time code

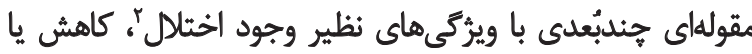

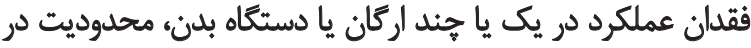
فعاليثها" و وجود موانعى براى مشاركت بـ فرد در جامعه.

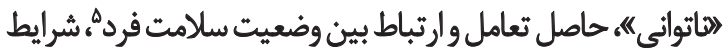

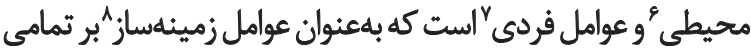

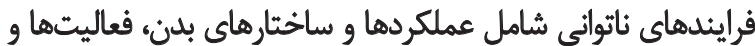

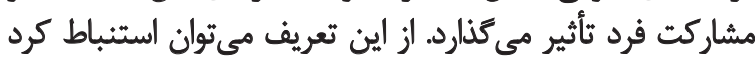

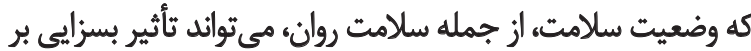
ناتوانى داشته باشد [مَّ]. با افزايش سن، شيوع برخى از اختلالات و ناتوانى هاى روانى افزايش

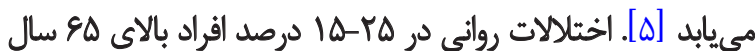

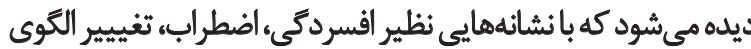
خواب، تنهاييى و انزواي اجتماعى همراه است [9.

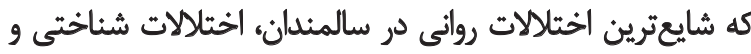
افسردكى هستند [V-9] و در بررسى هاتى مختلف شيوع اختئلاتلات

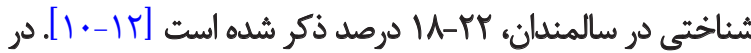

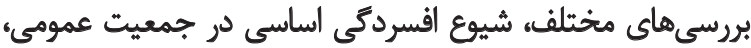

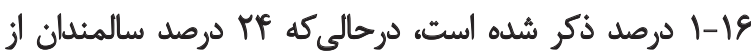

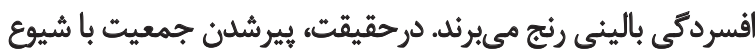
بيشتر افسردگى همراه است [9].

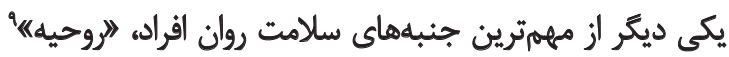

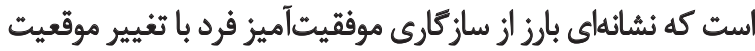

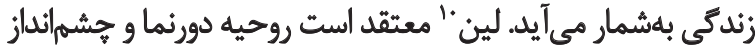

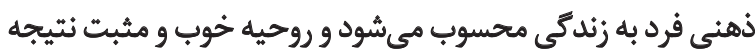

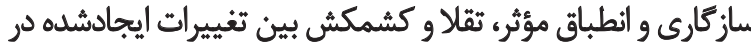

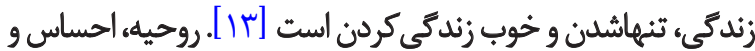

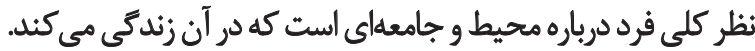
روحيه بهعنوان شاخص و تعيين كننده مفهوم رضايت از زندكى

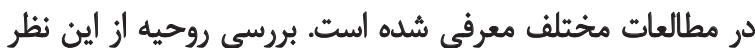

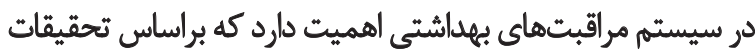
متعدد، اين مفهوم در تعامل كامل با سلامت است و ارتباط تنكاتئكى

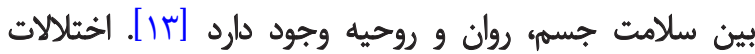
روانشناختى و افسردگى، منجر به ناتوانى افراد سالمند و ناتوانى،

2. Impairment

3. Activity limitation

4. Participation restriction

5. Health condition

6. Environment condition

7. Personal factors

8. Contextual factors

9. Morale

10. Lin 


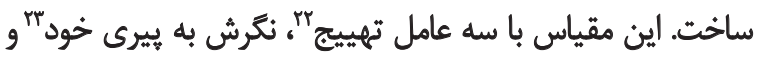

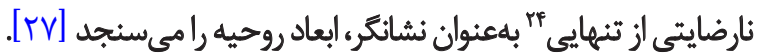

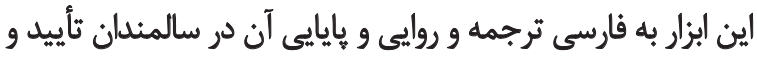

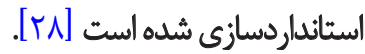

براي مقايسه متغير سن در بين زنان و مردان از آزمون تى مستقل

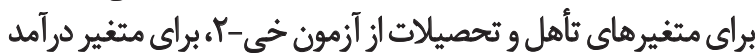

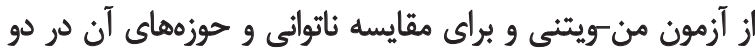

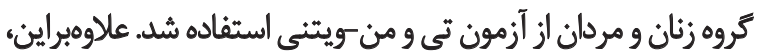

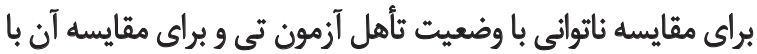

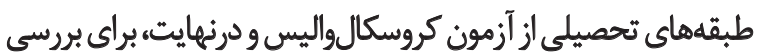

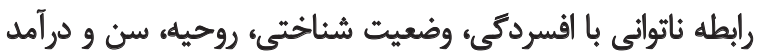

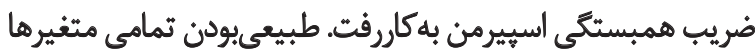

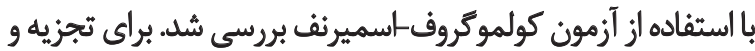

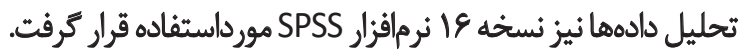

latiot

تعداد سالمندان دريافت كثنده خدمات از مركز روزانه سالمثدان

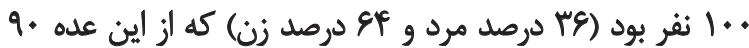

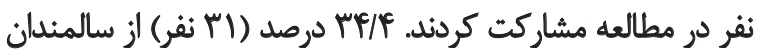

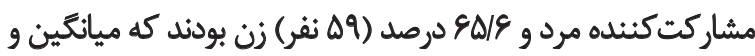
انحراف معيار سن آنان

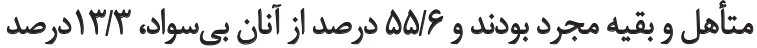

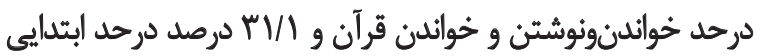

22. Agitation

23. Attitude toward qwn aging

24. Lonely dissatisfaction

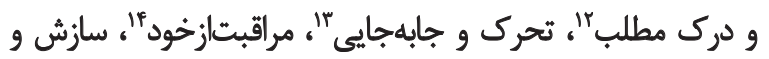

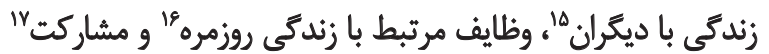

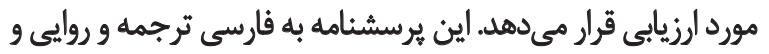

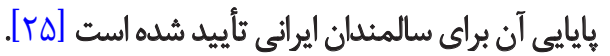
براى بررسى وضعيت شناختى از آزمون كوتاه شناختى ^استفاده

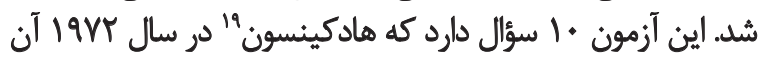

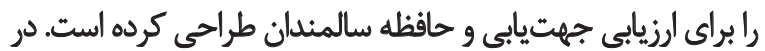

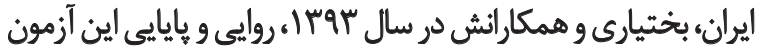
را بررسى و تأييد كردند [عبارئ.

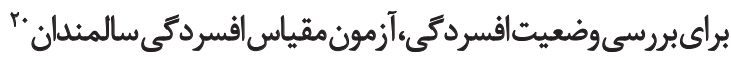
فرم كوتاه بهكارفت. اين آزمون توسط ملكوتى و همكار إنش دمر سال

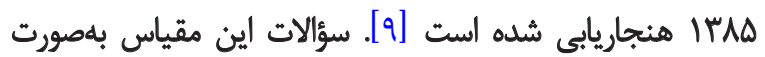

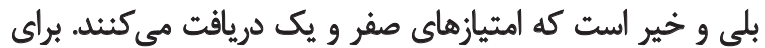

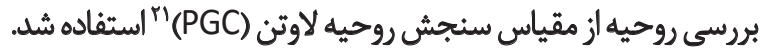

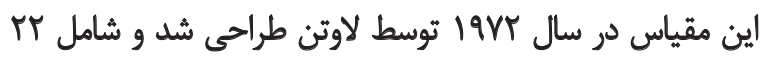

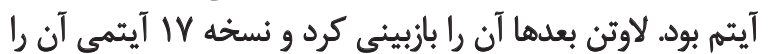
12. Understanding and communication
13. Getting around
14. Self care
15. Getting along with people
16. Life activities
17. Participation
18. Abreviated mental test
19. Hodkinson
20. Geriatric depression scale
21. Lawoton's PGC morale scale

جدول ا. مشخصات جمعيتشئاختي و همسائى زنان و مردان سالمند دريافت كئده خدمات از مركز روزانه سالميدان.

\begin{tabular}{|c|c|c|c|c|c|c|c|}
\hline الحتمال & آماره آزمون & كل & زنان & مردان & & & \\
\hline & & q. & $\Delta q$ & r & & & \\
\hline \multirow{2}{*}{$.1+.1$} & \multirow{2}{*}{$r M^{a}$} & & $V \cdot N E$ & $V \uparrow / \varepsilon_{\Delta}$ & & & \multirow{2}{*}{ سن } \\
\hline & & $9 / 80$ & ra & efre & أنحراف هعيار & & \\
\hline \multirow{2}{*}{$<+1+\infty 1$} & \multirow{2}{*}{$8 / /^{b}$} & $\Delta q(\varepsilon \Delta / 8)$ & $\Gamma \cdot(\Delta+/ \Lambda)$ & $r q(q m / \Delta)$ & $\%(n)$ & متأهل & \multirow{2}{*}{ تاهل } \\
\hline & & $M(M E / T)$ & $r q(p q / T)$ & $r(\theta / 0)$ & $\%(n)$ & مجرد & \\
\hline \multirow{3}{*}{$\cdot / A \cdot V$} & \multirow{3}{*}{.$/ \pi^{e b}$} & $\Delta \cdot(\Delta \Delta / F)$ & $M F(\Delta V / \varepsilon)$ & $\mid \gamma(\Delta V / q)$ & $\%(n)$ & بىسواد & \multirow{3}{*}{ تحصيلات } \\
\hline & & $M(\mid \Psi / T)$ & $N(\mid H / E)$ & $P(1 T / q)$ & $\%(n)$ & كمبسواد & \\
\hline & & rA(rY/I) & IV(TNA) & $\|(r \Delta / \Delta)$ & $\%(n)$ & باسواد & \\
\hline \multirow{2}{*}{.1 .50} & \multirow{2}{*}{$A P V / \Delta^{c}$} & T/fQ & TMA & Plea & & ميائكين & \multirow{2}{*}{ دروآمده } \\
\hline & & $r / f A$ & r/Tr & rffe. & & انحراف معيار & \\
\hline
\end{tabular}


جدول r. مقايسه ميانكين نمرات ناتواني، روحيه، افسردكي و وضعيت شناختي در مردان و زنان سالمند دريافت كثنده خدمات.

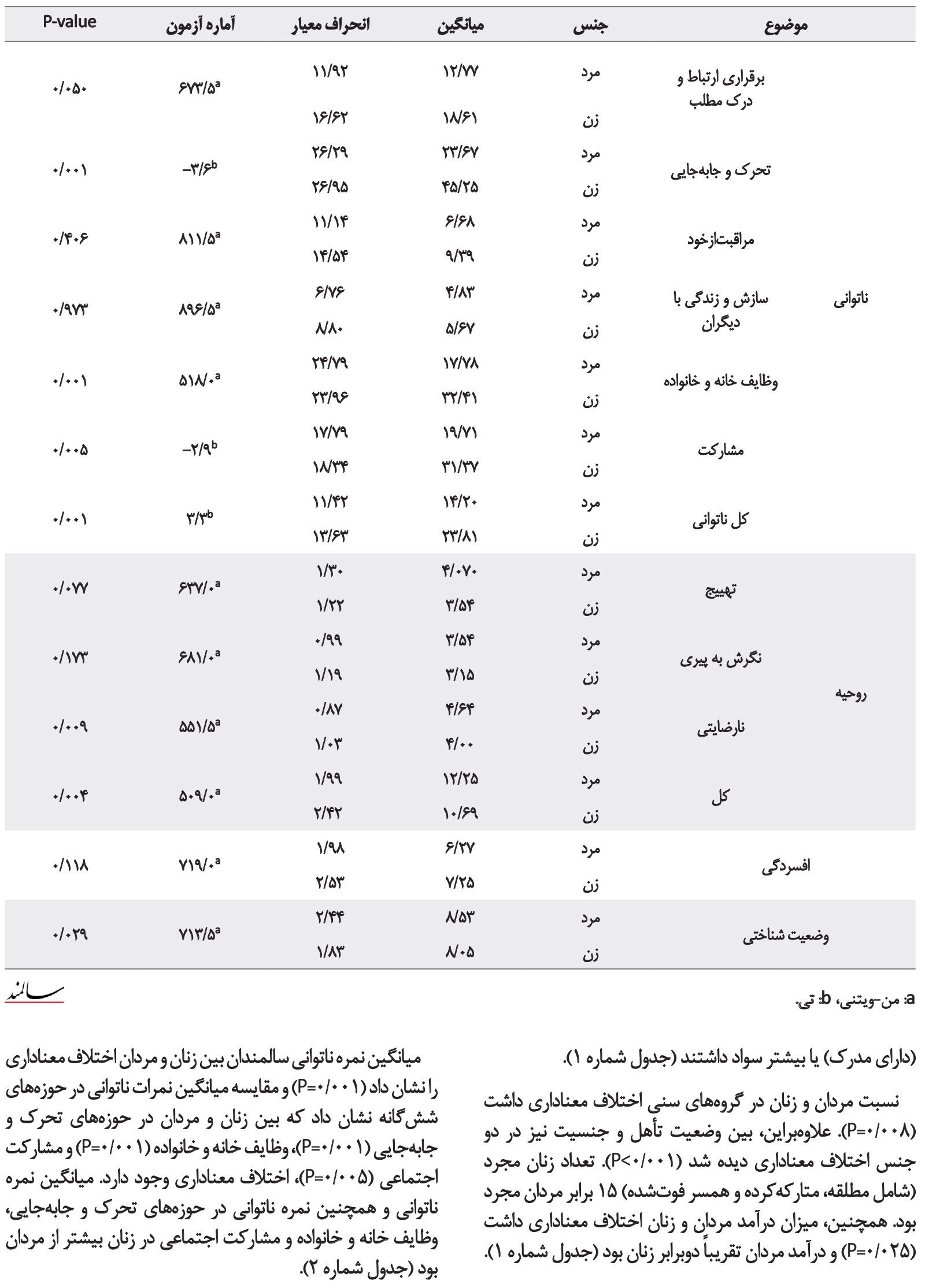


جدول ب. مقايسه ميانكين نمرات ناتوانى با جنسيت، وضعيت تأهل و تحصيلات سالمندان دريافت كننده خدمات.

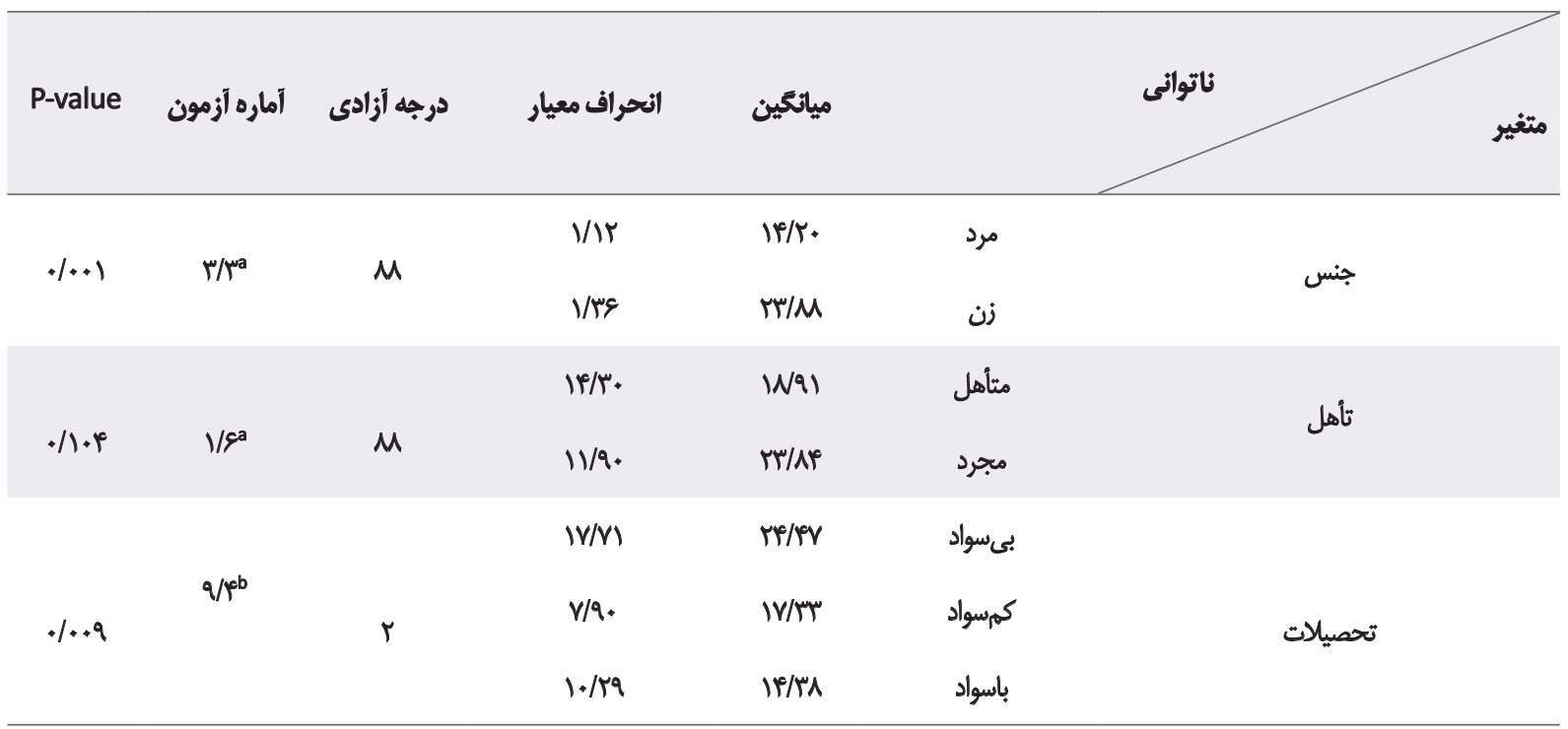

次

جدول F. ارتباط نمرات ناتوائى با افسردكى، وضعيت شئاختى، روحيه، سن و درآمد در سالمئدان دريافتكثئده خدمات.

\begin{tabular}{|c|c|c|c|}
\hline P-value & ضريب همبستكى اسييرمن & & متغير \\
\hline$<\cdot 1 . .1$ &.$|9|$ & & افسردكى \\
\hline$<\cdot 1 .+1$ &.$-|A|$ & & وضعيت شناختى \\
\hline$<\cdot 1 \cdot . \cdot 1$ & $-\operatorname{Mer}$ & تهييج & \\
\hline$<+1 .+1$ &.$- / 4$ & نكرش به ييرى & \\
\hline$<\cdot 1 \cdot+1$ &.$- / 4 t$ & نارضايتي & روحيه \\
\hline$<+1+.1$ & $-\cdot|8|$ & كل & \\
\hline איזr/. &. & & سن \\
\hline.$/ 94$. & $-.1 \cdot 1$ & & درآمد \\
\hline
\end{tabular}

il

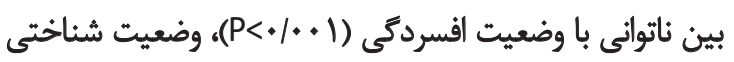

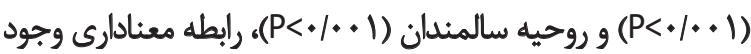

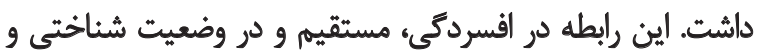

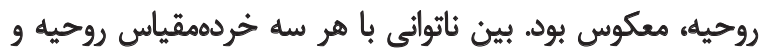

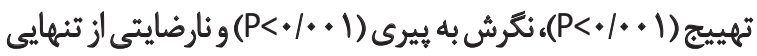

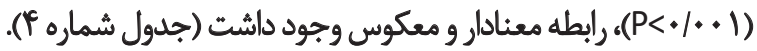

\&)

نتايج اين يُرهش نشان داد كه ناتوانى با وضعيت افسردكى،

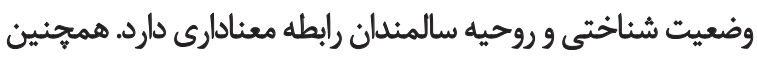

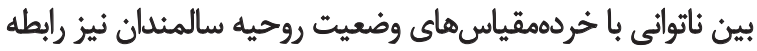

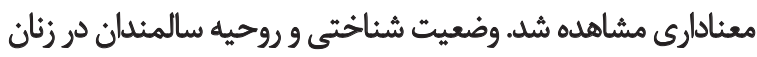

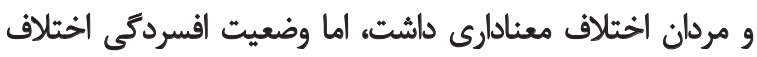

ميانكين نمرات ناتواني در زروههاي تحصيلي نيز اختّلاف معنادارى

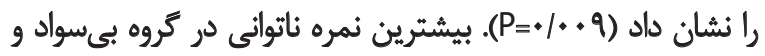

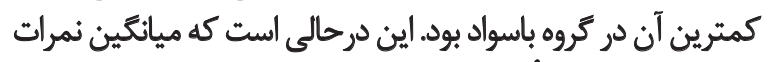

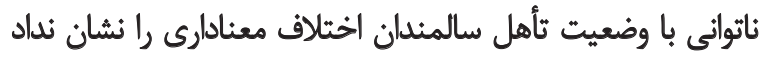

$$
\text { (جدول شماره باته). }
$$

در نمره روحيه، بين زنان و مردان اختّلاف معنادارى داشت رانت

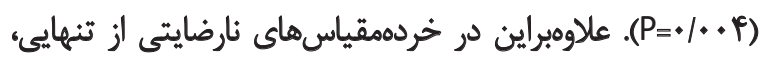

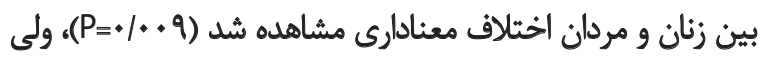

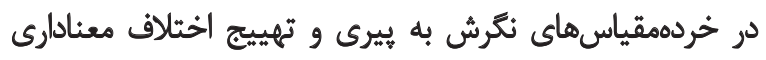

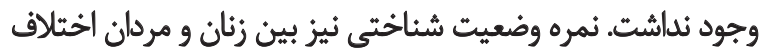

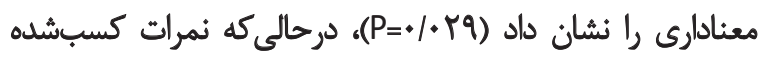
افسردگى، اختلاف معنادارى رانشان نداد (جدول شماره (Y). 


\section{نتيجهيَيرى نهايى}

با بتايج اين مطالعه نشان مي دهد كه بين ميزان ناتواني در سالمندان

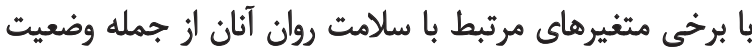

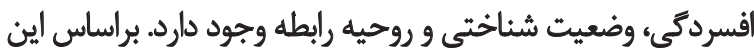
يافتهها مي توان موارد ذيل را توصيه نموديه

•انجام اقدامات بيشكَيرانه ودرمان بهموقع افسردگى در سالمنداني،

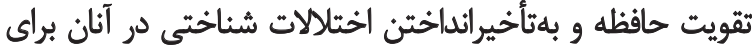
كمك به كاهش ميزان ناتوانى آنان؛

•اجراى برنامههاى تقويت روحيه، ايجاد نكّرش مثبت به بيرى و

$$
\text { تقويت رضايتمندى آنان از زندكىي؛ }
$$

• توسعه مراكز روزانه سالمندى و توسعه خدمات درمانى و

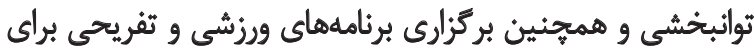

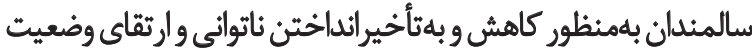

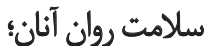

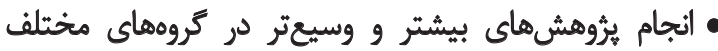
سالمندان براى بررسى دقيقتر رابطه ناتوانى بانئ با وضعيت سلامت

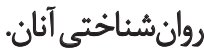

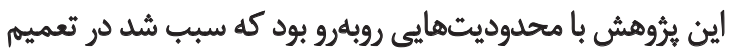

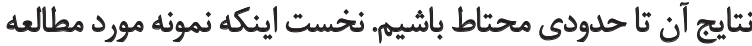

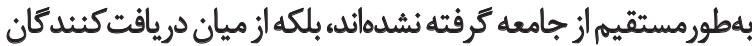

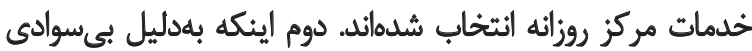

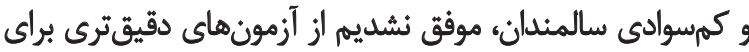
ارزيابى وضعيت شناختى سالمندان استفاده نماييم.

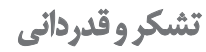

دريايان، يروهشكَران ازمديران و كاركنان آسايشَاه خيريه كهريزى استان البرز براى همكارى در انجام اين مطالعه كمال تشكر وقدان إنداني

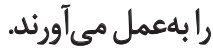

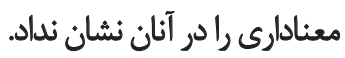

مقايسه ناتواني سالمندان در زنان و مردان، اختلاف معناداري داشت

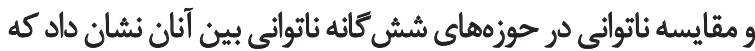

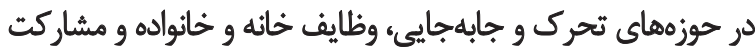
اجتماعى، بين زنان و مردان اختلاف معنادارى وجود داردان

همانطوركه قبلاً اشاره شد، بين ناتوانى سالمندان و وضعيت وانيت

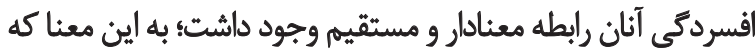

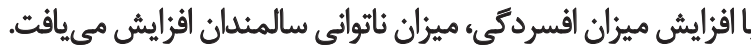

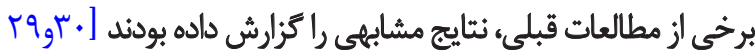

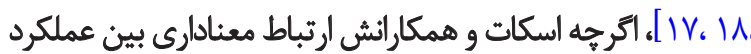

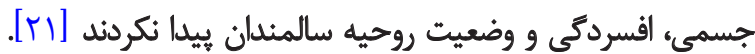

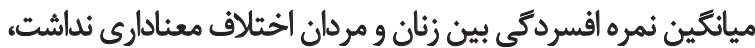

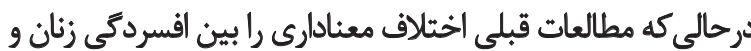

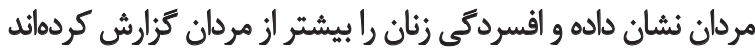
$\left.\left[q,{ }^{\prime}\right)\right]$

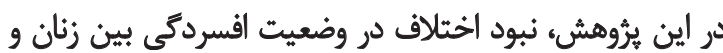

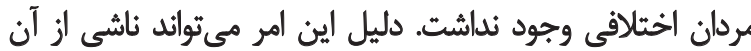

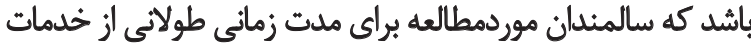

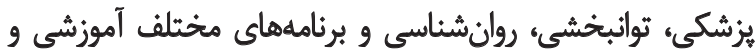

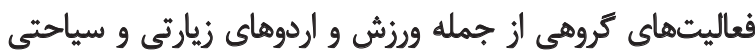

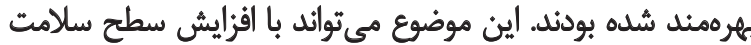

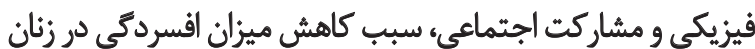

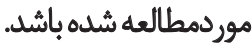

بين ناتوانى سالمندان و وضعيت شناختى آنان رابطه معنادار و وإمبات

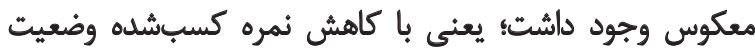

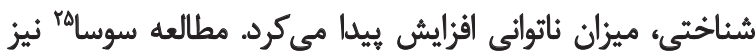

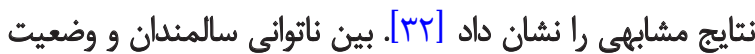

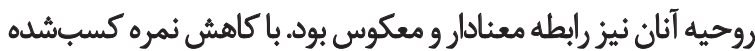

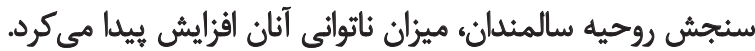

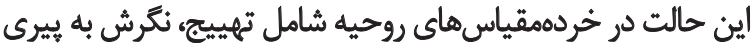

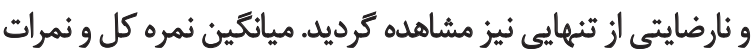

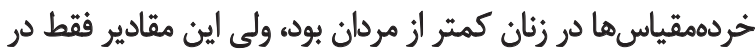

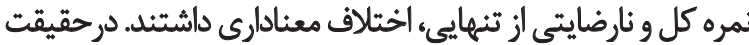

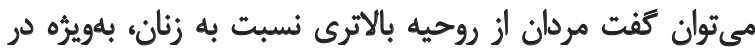

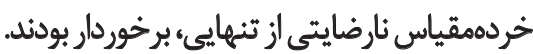
اين مطالعه نشان داد كه بيشترين ميزان ناتوانى سالمندان در

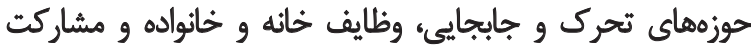

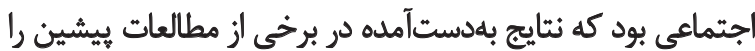

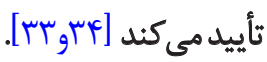




\section{References}

[1] Delavar B. [Elderly situation in Iran (Persian)]. Paper presented at: The $1^{\text {st }}$ Congress of Ageing Issues in Iran and Other Countries; 1999; Tehran, Iran.

[2] Ghaisarian A. [Assessment of social and economic dimensions of aging phenomena in Iran (Persian)]. Population. 2009; 69-70:127. Available from: www.sabteahval.ir/Upload/Modules/Contents/asset0/jma069-.pdf.

[3] Wilmoth JM, Ferraro KF. Gerontology: Perspectives and Issues. $4^{\text {th }}$ ed. New York: Springer; 2013.

[4] Minichiello V. Contemporary issues in gerontology. New York: Routledge Publishing; 2005.

[5] Najafi B, Arzaghi M, Fakhrzadeh H, Sharifi F, Shoaei S, Alizadeh $\mathrm{M}$, et al. [Mental health status and related factors in aged population: Urban health equity assessment and response tool (UrbanHeart) study in Tehran (Persian)]. Journal of Diabetes and Metabolism. 2013; 13(1):62-73.

[6] Nabavinejad H. [Mental health promoting models in elderly (Persian)]. The $1^{\text {st }}$ Congress of Ageing Issues in Iran and Other Countries; 1999; Tehran, Iran.

[7] Esperanza A, Miralles R, Rius I, Fernández B, Digon A, Arranz $\mathrm{P}$, et al. Evaluation of functional improvement in older patients with cognitive impairment, depression and/or delirium admitted to a geriatric convalescence hospitalization unit. Gorontology \& Geriatrics. 2004; 9:149-53.

[8] Nejati V, Ashayeri H. [Evaluation of relationship between depression and cognitive impairment in elderly (Persian)]. Iranian Journal of Aging. 2006; 1(2):112-18.

[9] Malakouti K, Fathollahi P, Mirabzadeh A, Salavati M, Kahani Sh. [Validation of geriatric depression scale (GDS-15) in Iran (Persian)]. Research in Medicine. 2006; 30(4):361-368.

[10] Kheirkhah F, Hoseini SR, Fallah R, Bijani A. [Prevalance of cognitive disorder in elderly people of Amirkola (2011-2012) (Persian)]. Iranian Journal of Psychiatry and Clinical Psychology. 2013; 19(4):247-254.

[11] Graham JE, Rockwood K, Beattie BL, Eastwood R, Gauthier S, Tuokko H, et al. Prevalence and severity of cognitive impairment with and without dementia in an elderly population. Lancet. 1997; 349(9068):1793-1796.

[12] Rait G, Fletcher A, Smeeth L, Brayne C, Stirling S, Nunes M, et al. Prevalence of cognitive impairment: Results from the MRC trial of assessment and management of older people in the community. Age and Ageing. 2005; 34(3):242-248

[13] Lin LC, Ou M, Wu SC. Factors influencing morale among the elderly in long-term care. Kaohsiung Journal of Medical Sciences. 1998;14(6):357-66.

[14] Donmez L, Gokkoca Z, Dedeoglu N. Disability and its effects on quality of life among older people living in Antalya city center, Turkey. Archives of Gerontology and Geriatrics. 2005; 40(2):213-23.

[15] Leonardi M, Raggi A, Pagani M, Carella F, Soliveri P, Albanese A, et al. Relationships between disability, quality of life and prevalence of nonmotor symptoms in Parkinson's disease. Parkinsonism \& Related Disorders. 2012; 18(1):35-9.
[16] Memarian R. [Self relainment and improving quality of life (Persian)] [Internet]. 1999. Available from: http://ganj.irandoc. ac.ir/articles/335259.

[17] Hashizume Y, Kanagawa K. Correlates of participation in adult Day care and quality of life in ambulatory frail elderly in Japan. Public Health Nursing. 1996; 13(6):404-415.

[18] Ara S, Foroughan M, Hatamizadeh N, Rahgozar M. [Evaluation of participation impact of elderly people in adult day centerKanoone Jahandidegan Fars center-on quality of life and general health of elderly people (Persian)] [MA thesis]. Tehran: University of Social Welfare and Rehabilitation Scinces; 2008.

[19] Arun M, Bharath S, Pal P, Singh G. Relationship of depression, disability, and quality of life in Parkinson's disease: A hospitalbased case-control study. Neurology India. 2011; 59(2):185-189.

[20] Banerjee A, Kumar S, Kulhara P, Gupta A. Prevalence of depression and its effect on disability in patients with age-related macular degeneration. Indian Journal of Ophthalmology. 2008; 56(6):469-474

[21] Scott KM, Von Korff M, Alonso J, Angermeyer MC, Bromet E, Fayyad J, et al. Mental-physical co-morbidity and its relationship with disability: Results from the world mental health surveys. Psychological Medicine. 2009; 39(1):33-43.

[22] Sousa RM, Ferri CP, Prince MJ. The relative impact of dementia on disability among older people in low and middle income countries. Alzheimer's and Dementia. 2008; 4(4):175-6.

[23] Conradsson M, Littbrand H, Bostrom G, Lindelof N, Gustafson $\mathrm{Y}$, Rosendahl E. Is a change in functional capacity or dependency in activities of daily living associated with a change in mental health among older people living in residential care facilities? Clinical Interventions in Aging. 2013; 8:1561-1568.

[24] Hadianfard M], Hadianfard H. [Mental Status of Geriatric Patients with Chronic Locomotor Diseases (Persian)]. Iranian Journal of Psychiatry and Clinical Psychology. 2004; 9(32):75-83.

[25] Arjmandhesabi M, Mahmoudi M, Kamali M, Zeraati H. [Translation and standardize of World Health Organization disability assessment schedule II- 36 items in Iranian elderly (Persian)] [MA thesis]. Tehran: University of Tehran; 2007.

[26] Bakhtiyari F, Foroughan M, Fakharzadeh H, Nazeri N, Najafi $\mathrm{B}$, Alizadeh $\mathrm{M}$, et al. [Validation of the persion version of abbreviated mental test (AMT) in elderly residents of Kahrizak charity foundation (Persian)]. Iranian Journal of Diabetes and Metabolism. 2014; 13(6):487-494.

[27] Lawton M. Lawton's PGC morale scale. Gerontology. 1975; 30(1):85-89.

[28] Foroughan M, Shakeri F, Farzad V. P478: Psychometrical featurs of Philadelphia Morale Scale in Iranian elderly. European Geriatrics Medicine. 2014; 5(1):233.

[29] Penninx BW, Leveille S, Ferrucci L, Van Eijk JT, Guralnik JM. Exploring the effect of depression on physical disability: Longitudinal evidence from the established populations for epidemiologic studies of the elderly. American Journal of Public Health. 1999; 89(9):1346-1352.

[30] Beekman AT, Penninx BW, Deeg DJ, Beurs ED, Geerlings SW, Tilburg WV. The impact of depression on the well-being, disability and use of services in older adults: A longitudinal perspective. Acta Psychiatrica Scandinavica. 2002; 105(1):20-27. 
[31] Kessler RC, McGonagle KA, Swartz M, Blazer DG, Nelson CB. Sex and depression in the National Comorbidity Survey I: Lifetime prevalence, chronicity and recurrence. Journal of Affective Disorders. 1993; 29(2-3):85-96.

[32] Sousa RM, Dewey ME, Acosta D, Jotheeswaran AT, CastroCosta E, Ferri CP, et al. Measuring disability across cultures - The psychometric properties of the WHODAS II in older people from seven low and middle-income countries: The 10/66 Dementia research Group population-based survey. International Journal of Methods in Psychiatric Research. 2010; 19(1):1-7.

[33] Shahbazi MR, Mirkhani M, Hatamizadeh N, Rahgozar M. [Disability Assessment in elderly people in Tehran city (Persian)]. Iranian Journal of Ageing. 2008; 3(3-4):84-92.

[34] Adib Hajbagheri M. [Geriatric Disability Related Factors (Persian)]. Iranian Journal of Ageing. 2008; 3(2):547-555.

[35] World Health Organization. International classification of functioning, disability and health. Geneva: Word Health Organization Publication; 2002. 
